\title{
On the universality class of a magnetic liquid in an external field
}

\author{
R. Folk ${ }^{11}$, G. Mose ${ }^{2]}$ \\ ${ }^{1}$ Institute for Theoretical Physics, Johannes Kepler University, Altenberger Str. 69, A-4040 Linz, Austria \\ 2 Department for Material Research and Physics, Paris Lodron University Salzburg, Hellbrunnerstrasse 34, \\ A-5020 Salzburg, Austria
}

Received February 9, 2010, in final form February 25, 2010

\begin{abstract}
Time scale ratios play an important role in critical dynamics. Their fixed point values govern the dynamical scaling behavior of the relevant fields and their dynamic correlation functions as well as the hydrodynamic transport coefficients. As an example for a system with several time scale ratios we consider a magnetic liquid near the gas-liquid transition.
\end{abstract}

Key words: phase transition, critical dynamics

PACS: $64.60 . H t, 05.70 . J k, 75.50 . \mathrm{Mm}, 64.70 . \mathrm{FX}$

\section{Introduction}

Collective modes in simple fluids, binary and ternary mixtures and more complex systems like magnetic or ionic fluids have been of interest for I. Mryglod over a long time in his scientific carrier (see his publication list). It is a pleasure for one of us (R.F.) having the chance to participate in this field with him over several years. Many aspects of such systems have been studied with different methods to get a more or less complete picture of the model and to understand the behavior of real systems [1-7]. The phase diagram of a magnetic liquid may show different topologies. The topology do change if such a liquid is exposed to an external magnetic field and as a result a line of gas-liquid critical points might exist [8 $[10]$.

In the following the critical dynamics of a magnetic fluid in an external magnetic field is discussed. After some general remarks concerning time scales and their ratios appearing in critical dynamics, the steps for defining the equation of motion near the gas-liquid critical point are indicated. As a conclusion one finds that the critical dynamics of the magnetic fluid can be mapped on the critical dynamics of a mixture. Taking this result into consideration the consequences for the critical dynamics and the behavior of the transport coefficients are discussed.

\section{General considerations on dynamics}

The critical dynamics is described by the dynamics of the slow density which are the order parameter density and the densities of conserved variables. The order parameter might be conserved or non-conserved. In any case its dynamics is slow due to a critical slowdown when the transition is approached. Each slow density has its own time scale set by the relaxation or diffusion coefficients appearing in the dynamic equations and driving the system to its thermodynamic equilibrium. Time scale ratios are then defined by the ratio of these kinetic coefficients. The question arises which densities have to be taken into account in order to define the universality class of the critical dynamical behavior. E.g., the densities describing the sound mode are conserved but the coupling of the sound mode to the dynamics of the order parameter equation has no effect on the genuine dynamical critical behavior of the order parameter. This is argued by considering the sound mode as faster than the diffusive modes defining the dynamical universality class of the order parameter. 
Dynamic scaling or more precisely strong dynamic scaling is realized when all time scales present in the dynamic model describing critical dynamics are related by finite nonzero time scale ratios. In consequence the critical dynamics is described by one time scale. This time scale may be defined by the characteristic frequency of the order parameter $\omega_{\mathrm{OP}}(k)=A_{\mathrm{OP}} k^{z_{\mathrm{OP}}}$ at the transition temperature $T_{\mathrm{c}}$. This is given by a power law in the wave vector modulus characterized by the dynamic critical exponent $z_{\mathrm{OP}}$. The amplitude $A$ is proportional to the relaxation or diffusion coefficient of the order parameter. The dynamical critical exponent $z_{\mathrm{OP}}$ is calculated in field theory from the so-called $\zeta$-function at the dynamical fixed point. The renormalization procedure, which removes singularities (for a general introduction see [11]), leads to a renormalized kinetic coefficient $\Gamma$ in the equation of motion for the order parameter. From these renormalization factors, the $\zeta$-functions are obtained and their values at the stable dynamic fixed point (characterized by a star) give the values of dynamical exponents. For the order parameter this reads

$$
z_{\mathrm{OP}}=2+\zeta_{\Gamma}^{\star}
$$

One approach to describe the slow dynamics is by using Langevin equations for the dynamical variables. The simplest situation may arise when the order parameter is non-conserved and there exists an additional conserved density. Such a situation may arise for an anisotropic (uniaxial) antiferromagnet in an external field where the order parameter is given by the staggered magnetization and the conserved density by the magnetization both in the direction of the external field [12, 13], which is an example for the so-called model $\mathrm{C}$ [14, 15]. In such a case an additional kinetic coefficient $\lambda$ appears in the equation for the conserved density.Thus a second characteristic frequency $\omega_{\mathrm{CD}}(k)=A_{\mathrm{CD}} k^{z_{\mathrm{CD}}}$ might be defined. In addition a second dynamical critical exponent might be defined by the corresponding $\zeta$-function for the conserved density

$$
z_{\mathrm{CD}}=2+\zeta_{\lambda}^{\star}
$$

The fixed point value of the time scale ratio $w$ of the two kinetic coefficients

$$
w=\frac{\Gamma}{\lambda}
$$

is found according to its definition from the stationary solution of the renormalization equation

$$
\ell \frac{\mathrm{d} w}{\mathrm{~d} \ell}=w(\ell)\left(\zeta_{\Gamma}(w(\ell), \ldots)-\zeta_{\lambda}(w(\ell), \ldots)\right)
$$

The dots indicate other possible renormalizing parameters of the dynamical equations. If the fixed point value of $w$ is different from zero and finite, the right hand side of (4) has to be zero. This immediately leads to the relation

$$
\zeta_{\Gamma}^{\star}=\zeta_{\lambda}^{\star}
$$

and consequently to only one dynamic critical exponent $z=z_{\mathrm{OP}}=z_{\mathrm{CD}}$, strong scaling is valid. If however the two exponents are different and the two kinetic coefficients scale differently, two time scales are present, the situation being called weak dynamic scaling. Such a situation arises for the anisotropic antiferromagnet at higher values for the external field. The dynamics belongs the to the universality class of model E [16] or if non-asymptotic effects are taken into account to model $\mathrm{F}$ [16].

A different situation arises in cases where the order parameter is already a conserved density. Then the time scale ratio to another conserved density is an irrelevant parameter since its naive dimension is e.g. -2 , whereas if the order parameter is non-conserved, the naive dimension of the time scales is zero. The first case arises at the gas-liquid critical point in a fluid where the order parameter, the entropy density (or a linear combination with the mass density), is conserved and dynamically couples with the transverse momentum current $\vec{j}_{\mathrm{t}}$. These two densities describe the critical modes of heat diffusion and the shear mode. Then two time scales are present from the beginning and two exponents describing the divergence of the thermal conductivity and the weak divergence of the shear viscosity have to be calculated. The time scale ratio has to be set to zero in 
the minimal subtraction scheme used to calculate the dynamical exponents [17]. The second case arises in the above mentioned model $\mathrm{C}$.

As already mentioned above a similar situation arises for the densities describing the sound mode when the minimal subtraction scheme is used [18, 19]. There the unrenormalized coupling constant between the density and the longitudinal part of the momentum current has to go to infinity. This procedure ensures that even the non-asymptotic critical dynamics of the order parameter is not effected by the sound mode and, on the other hand, the critical effect in the sound mode can be calculated.

In what follows, the critical dynamics of a magnetic fluid in an external magnetic field is considered and the steps leading to the dynamical model describing its critical dynamics are presented. It turns out 20] that the critical dynamics belongs to the universality class of a pure fluid [21] (model H [16]), whereas the non-asymptotic behavior belongs to the case of a mixture [19, 22].

\section{Hydrodynamics of Heisenberg fluids in an external magnetic field}

Magnetic fluids are well known systems combining translational and magnetic degrees of freedom. A subclass of such systems are those magnetic liquids where on the microscopic level the magnetic moments (spins) interact via a short ranged Heisenberg interaction [23 25]. In order to obtain the hydrodynamic equations for a Heisenberg fluid in a magnetic field $\vec{H}_{\text {ex }}$ we have to consider the corresponding slow extensive densities per volume near the gas liquid critical point. These are first of all those densities already describing the critical dynamics of a simple liquid, namely the entropy density $s(x)$, the mass density $\rho(x)$ and the momentum density $\vec{j}(x)$, which is a vector in the coordinate space with components $j_{i}(x)(i=x, y, z)$. Additionally, one has to consider the components of a classical spin density $m^{\mu}(x)$, where $\mu=x, y, z$ denotes the components in spin space, describing the magnetic degrees of freedom in the direction of the external field. Thus, independent of the nature of the spin, either Ising, XY or Heisenberg, only one component is involved in the dynamics. An intensive conjugate field corresponds to each density. These fields are the temperature $T(x)$, the chemical potential $\mu(x)$, the velocity $\vec{v}(x)$ and the magnetic field $\vec{H}(x)$. The introduced densities define four hydrodynamical modes of the system: the thermal diffusion, the shear mode, the sound mode and the spin diffusion.

The reversible Euler equations for the densities result from generalized Poisson bracket relations and a corresponding "Hamiltonian" $\mathcal{H}$. Denoting the extensive densities per volume generally with $\alpha_{i}(x)$, the reversible equations have the form

$$
\frac{\partial \alpha_{i}(x)}{\partial t}=\left\{\alpha_{i}(x), \mathcal{H}\right\}
$$

The "Hamiltonian" in the present macroscopic case is effectively a static functional $\mathcal{H}=\int \mathrm{d}^{3} x e\left(\left\{\alpha_{i}(x)\right\}\right)$. The whole ensemble $\left\{\alpha_{i}(x)\right\}$ consists of the extensive densities $\alpha_{i}(x)$. The total energy density

$$
e=u+e_{\mathrm{kin}}-\sum_{\mu} H_{\mathrm{ex}}^{\mu} m^{\mu}
$$

of the system is composed of the internal energy $u$, where

$$
\mathrm{d} u=T \mathrm{~d} s+\mu \mathrm{d} \rho+\sum_{\mu} H^{\mu} \mathrm{d} m^{\mu},
$$

the kinetic energy $e_{\mathrm{kin}}=\frac{1}{2} \rho \vec{v}^{2}$ and the energy of the spin density in the external field $-\sum_{\mu} H_{\mathrm{ex}}^{\mu} m^{\mu}$. The magnetic field $H^{\mu}=\left(\frac{\partial u}{\partial m^{\mu}}\right)_{s, \rho, \vec{j}}$ represents the internal magnetic field produced by the spin density.

Following [26], the generalized Poisson brackets $\left\{\alpha_{i}, \alpha_{j}\right\}$ between the conserved densities are determined by the group generators of operations in coordinate and spin space. The momentum 
density, as the generator of translations in coordinate space, determines the Poisson brackets,

$$
\begin{aligned}
\left\{s(x), \vec{j}\left(x^{\prime}\right)\right\} & =s\left(x^{\prime}\right) \vec{\nabla}^{\prime} \delta\left(x-x^{\prime}\right), \\
\left\{\rho(x), \vec{j}\left(x^{\prime}\right)\right\} & =\rho\left(x^{\prime}\right) \vec{\nabla}^{\prime} \delta\left(x-x^{\prime}\right), \\
\left\{j_{i}(x), j_{k}\left(x^{\prime}\right)\right\} & =j_{i}\left(x^{\prime}\right) \nabla_{k}^{\prime} \delta\left(x-x^{\prime}\right)-j_{k}(x) \nabla_{i} \delta\left(x-x^{\prime}\right),
\end{aligned}
$$

where the prime on $\vec{\nabla}^{\prime}$ indicates that it acts on $x^{\prime}$. In quantum mechanics, the spin operator is the generator of rotations in spin space, acting only on the spin components rather than on quantities in coordinate space. The components of the spin density are introduced as $m^{\mu}(x)=\sum_{i} S_{i}^{\mu} \delta\left(x-x_{i}\right)$. In a coarse-grained theory the $S_{i}^{\mu}$ represent the mean $\mu$-component of the spin in a small volume located at $x_{i}$. The Poisson bracket generated by the spin rotation is

$$
\left\{m^{\mu}(x), m^{\nu}\left(x^{\prime}\right)\right\}=-\sum_{\lambda} \epsilon^{\mu \nu \lambda} m^{\lambda}(x) \delta\left(x-x^{\prime}\right)
$$

The spin components $S_{i}^{\mu}$ are not affected by transformations in coordinate space such as translation or rotation. However, the spin density $m^{\mu}(x)$ is affected by such transformations due to the scalar $\delta$ function. It generates a Poisson bracket between the magnetization $m$ and the momentum density, which has the same structure as for the entropy density

$$
\left\{m^{\mu}(x), \vec{j}\left(x^{\prime}\right)\right\}=m^{\mu}\left(x^{\prime}\right) \vec{\nabla}^{\prime} \delta\left(x-x^{\prime}\right) .
$$

Then the following Euler equations are obtained

$$
\begin{aligned}
\frac{\partial s}{\partial t}+\vec{\nabla} \cdot(s \vec{v}) & =0, \\
\frac{\partial \rho}{\partial t}+\vec{\nabla} \cdot(\rho \vec{v}) & =0, \\
\frac{\partial j_{i}}{\partial t}+\nabla_{i} P+\sum_{l} \nabla_{l} v_{l} j_{i} & =0, \\
\frac{\partial m^{\mu}}{\partial t}+\epsilon^{\mu \nu \lambda}\left(H^{\nu}-H_{\mathrm{ex}}^{\nu}\right) m^{\lambda}+\sum_{l} \nabla_{l} v_{l} m^{\mu} & =0 .
\end{aligned}
$$

So far, equations (14)-(17) do not contain dissipative processes. We have made use of the fact that the external field is homogeneous, otherwise an additional term proportional to the scalar product between the magnetisation and the gradient of the field appears. This would considerably change the resulting critical model and is not discussed here. In fact, it would lead to anisotropies in the fluid system [27].

The total energy $\mathcal{H}$ has to remain constant in time, i.e. $\partial \mathcal{H} / \partial t=\int \mathrm{d}^{3} x(\partial e / \partial t)=0$, which can be immediately verified by using the continuity equations from above. Using the relation $\vec{j}(x)=\rho(x) \vec{v}(x)$ and introducing the tensor $[\boldsymbol{P}]_{k i} \equiv P_{k i}=P \delta_{k i}$, one immediately obtains the equation for the velocity $\vec{v}$ from the conservation equations for momentum density (16), and finally the hydrodynamic equation for the kinetic energy

$$
\frac{\partial e_{\mathrm{kin}}}{\partial t}=-\vec{\nabla} \cdot\left[e_{\mathrm{kin}} \vec{v}+\boldsymbol{P} \cdot \vec{v}\right]+\sum_{i, k} P_{k i} \nabla_{k} v_{i}
$$

The first term on the right hand side in (18) represents the divergence of the kinetic energy current $e_{\text {kin }} \vec{v}+\boldsymbol{P} \cdot \vec{v}$. In addition, an energy source term appears in (18) because the kinetic energy is not a conserved density. The total energy density $e$ is conserved and therefore obeys the continuity equation,

$$
\frac{\partial e}{\partial t}=-\vec{\nabla} \cdot \overrightarrow{J_{\mathrm{e}}}
$$

where $\overrightarrow{J_{\mathrm{e}}}$ is the total energy current. Without dissipative effects, the total energy current is the sum of the convective flow $e \vec{v}$ and the flow of mechanical work $\boldsymbol{P} \cdot \vec{v}$. 
In order to treat dissipation we have to append the dissipative currents [28] to equations (14), (16), (17) and (19). The total energy current contains two dissipative processes. The first of these accounts for the finite viscosity of the fluid. Viscosity causes additional mechanical work when different fluid layers shift against each other, and is taken into account using an extension of the pressure tensor,

$$
P_{k i}=P \delta_{k i}+\Pi_{k i},
$$

where $\Pi_{k i}$ is a symmetric tensor. The second dissipative process takes into account the energy transport by heat conduction, which may be included by adding a heat current $\vec{J}_{\mathrm{q}}$ to the total energy current. Thus we get

$$
\overrightarrow{J_{\mathrm{e}}}=e \vec{v}+\boldsymbol{P} \cdot \vec{v}+\vec{J}_{\mathrm{q}}
$$

for the total energy current with dissipation. The contribution of the Heisenberg interaction to the energy of a spin system depends only on the orientation of the spins. Therefore the spin density does not contribute to the energy transport and in (21) no magnetic current is present.

Since in the following we will consider a fluid in an external magnetic field $\vec{H}_{\mathrm{ex}}=H_{\mathrm{ex}} \vec{e}_{z}$, with $\vec{e}_{z}$ being the unit vector in the $z$-direction in the spin space. Thus only the $z$ component $m^{z}(x)$ of the spin density is conserved. From (17) it follows that the $z$-component now fulfills the equation

$$
\frac{\partial m^{z}}{\partial t}+\sum_{l} \nabla_{l} v_{l} m^{z}=-\vec{\nabla} \vec{J}_{m}^{z}
$$

where $\vec{J}_{m}^{z}$ is the spin current vector.

Inserting equation (20) into the equation for the momentum current equations (16)

$$
\frac{\partial j_{i}}{\partial t}+\nabla_{i} P+\sum_{l} \nabla_{l} v_{l} j_{i}=-\sum_{l} \nabla_{l} \Pi_{l i}^{(\mathrm{s})}-\nabla_{i} \Pi
$$

the dissipative processes are included. In the above equation, the symmetric tensor $[\Pi]_{i k}=\Pi_{i k}=$ $\Pi_{i k}^{(\mathrm{s})}+\Pi \delta_{i k}$ introduced in (20) has been separated into the traceless contribution $\left[\boldsymbol{\Pi}^{(\mathrm{s})}\right]_{i k}=\Pi_{i k}^{(\mathrm{s})}$ and the trace $\Pi=\frac{1}{3} \sum_{l} \Pi_{l l}$.

The dissipative current in the entropy equation is found from the dissipative currents already introduced in the energy, the magnetic density and the momentum current. From (8) we also determine the equation for the entropy density conservation

$$
T \frac{\partial s}{\partial t}=\frac{\partial u}{\partial t}-\sum_{\mu} H^{\mu} \frac{\partial m^{\mu}}{\partial t} .
$$

From (7) we obtain the hydrodynamic equation for the internal energy $u$

$$
\frac{\partial u}{\partial t}=\frac{\partial e}{\partial t}-\frac{\partial e_{\mathrm{kin}}}{\partial t}+\sum_{\mu} H_{\mathrm{ex}}^{\mu} \frac{\partial m^{\mu}}{\partial t} .
$$

Inserting (19), (18) and (22) we obtain for the internal energy the equation,

$$
\frac{\partial u}{\partial t}=-\vec{\nabla} \cdot\left[u \vec{v}+\vec{J}_{\mathrm{q}}\right]-\sum_{i, k} P_{k i} \nabla_{k} v_{i}-\sum_{\mu, \nu, \lambda} \epsilon^{\mu \nu \lambda} H^{\mu} m^{\nu} H_{\mathrm{ex}}^{\lambda} .
$$

The final equation for the entropy density is found by inserting (26) into (24)

$$
\frac{\partial s}{\partial t}+\vec{\nabla} \cdot(s \vec{v})=-\vec{\nabla} \cdot\left(\frac{\vec{J}_{\mathrm{q}}-\vec{J}_{m}^{z} H^{z}}{T}\right)+q_{\mathrm{s}}
$$

with $q_{\mathrm{s}}$ the entropy source term,

$$
q_{\mathrm{s}}=-\left(\vec{J}_{\mathrm{q}}-\vec{J}_{m}^{z} H^{z}\right) \cdot \frac{\vec{\nabla} T}{T^{2}}-\vec{J}_{m}^{z} \cdot \frac{\vec{\nabla} H^{z}}{T}-\Pi^{(\mathrm{s})}: \frac{\boldsymbol{\nabla} \boldsymbol{v}^{(\mathrm{s})}}{T}-\Pi \frac{\vec{\nabla} \cdot \vec{v}}{T} .
$$


Some notations of tensor algebra have been introduced in the above relation, namely $\boldsymbol{a} \boldsymbol{b}$ denotes the dyadic product $[\boldsymbol{a} \boldsymbol{b}]_{i j}=a_{i} b_{j}$ of two vectors $\vec{a}$ and $\vec{b}$. The symbol colon ':' expresses the total contraction of two tensors of the second rank, $\boldsymbol{A}: \boldsymbol{B}=\sum_{i, j} A_{i j} B_{i j}$.

From (28) we can see that the entropy source has the structure [29],

$$
q_{\mathrm{s}}=-\sum_{i} J_{i} X_{i}
$$

with the currents $J_{i}$ and corresponding thermodynamic forces $X_{i}$. In the present case the thermodynamic forces are $\vec{\nabla} \cdot \vec{v} / T, \vec{\nabla} T / T^{2}, \vec{\nabla} H^{z} / T$ and $\nabla \boldsymbol{v}^{(\mathrm{s})} / T$ The thermodynamic forces have different tensor characters. The first one is a scalar, the second and the third are polar vectors, the fourth is a polar tensor of the second rank. The same is true for the currents. The currents $J_{i}=J_{i}\left(\left\{X_{j}\right\}\right)$ are functions of the thermodynamic forces. For small gradients we can assume a linear dependence $J_{i}=\sum_{j} L_{i j} X_{j}$, where $L_{i j}$ are the Onsager coefficients. Generally, all currents may depend on all thermodynamic forces that define a set of Onsager coefficients, which, of course, have different tensor character depending on the tensor character of the connected current and force. But the interaction symmetry and the tensor character of thermodynamic forces restrict the form of thermodynamic forces in a current and reduces the number of non-vanishing Onsager coefficients. For an isotropic system, the form of thermodynamic forces follows from invariance under coordinate inversion and arbitrary rotation, so that only tensor characters of the same type can have cross coefficients. In (28) two polar vectors $\vec{\nabla} T / T^{2}$ as well as $\vec{\nabla} H^{z} / T$ are present. Thus, the resulting currents are,

$$
\begin{aligned}
\vec{J}_{\mathrm{q}}-\vec{J}_{m}^{z} H^{z} & =-\gamma \vec{\nabla} T-T \beta \vec{\nabla} H^{z} \\
\vec{J}_{m}^{z} & =-\beta \vec{\nabla} T-\alpha \vec{\nabla} H^{z} \\
\boldsymbol{\Pi} & =-2 \bar{\eta} \boldsymbol{\nabla} \boldsymbol{v}^{(\mathrm{s})} \\
\Pi & =-\zeta \vec{\nabla} \cdot \vec{v} .
\end{aligned}
$$

In equations (30) and (31) we have already used the symmetry properties of the Onsager coefficients. Linearizing the hydrodynamic equations about the mean values $\bar{s}, \bar{m}^{z}, \bar{\rho}$ and $\vec{j}=0$ we obtain,

$$
\begin{aligned}
\frac{\partial s}{\partial t} & +\bar{s} \vec{\nabla} \cdot \vec{v}=\frac{\gamma}{T} \nabla^{2} T+\beta \nabla^{2} H^{z}, \\
\frac{\partial m^{z}}{\partial t} & +\quad \bar{m}^{z} \vec{\nabla} \cdot \vec{v}=\beta \nabla^{2} T+\alpha \nabla^{2} H^{z}, \\
\frac{\partial \rho}{\partial t} & +\bar{\rho} \vec{\nabla} \cdot \vec{v}=0, \\
\frac{\partial \vec{j}}{\partial t} & +\quad \vec{\nabla} P=\bar{\eta} \nabla^{2} \vec{v}+\left(\zeta+\frac{4}{3} \bar{\eta}\right) \vec{\nabla}(\vec{\nabla} \cdot \vec{v}) .
\end{aligned}
$$

A statistical treatment of generalized hydrodynamic equations can be found in [30].

The Onsager coefficients $\alpha, \beta$ and $\gamma$ introduced in (30) and (31) can be related to the thermal conductivity $\kappa_{\mathrm{T}}$ or the thermal diffusion coefficient $D_{\mathrm{T}}$ respectively, the thermomagnetic diffusion ratio $k_{\mathrm{T}}$ and the spin diffusion coefficient $D_{m}$. The coefficients $\bar{\eta}$ and $\zeta$ represent the shear and bulk viscosity. Assuming a vanishing spin current $\vec{J}_{m}^{z}=0$ in (31), the gradients of the temperature and magnetic field are related by $\vec{\nabla} H^{z}=-(\beta / \alpha) \vec{\nabla} T$. Inserting this relation into (30), we obtain for the heat current

$$
\overrightarrow{J_{\mathrm{q}}}=-\left(\gamma-\frac{T \beta^{2}}{\alpha}\right) \vec{\nabla} T,
$$

from which we identify the thermal conductivity $\kappa_{\mathrm{T}}$ at vanishing spin current to be,

$$
\kappa_{\mathrm{T}}=\gamma-\frac{T \beta^{2}}{\alpha} .
$$


At constant spin density per mass $\mu_{m}=m^{z} / \rho$ and constant pressure $P$, the temperature gradient is proportional to the gradient of the entropy density per unit mass $\sigma=s / \rho$,

$$
\vec{\nabla} T=\left(\frac{\partial T}{\partial \sigma}\right)_{\mu_{m}, P} \vec{\nabla} \sigma
$$

The equation for $\sigma$ is easily derived from equations (34) and (36),

$$
\frac{\partial \sigma}{\partial t}=\frac{\kappa_{\mathrm{T}}}{\rho T}\left(\frac{\partial T}{\partial \sigma}\right)_{\mu_{m}, P} \nabla^{2} \sigma
$$

and this describes heat conduction in the magnetic fluid. From this equation we identify the thermal diffusion coefficient to be

$$
D_{\mathrm{T}}=\frac{\kappa_{\mathrm{T}}}{\rho T}\left(\frac{\partial T}{\partial \sigma}\right)_{\mu_{m}, P} .
$$

Generally, in the case of local thermodynamic equilibrium the gradient of the internal magnetic field at constant pressure can be written as

$$
\vec{\nabla} H^{z}=\left(\frac{\partial H^{z}}{\partial T}\right)_{m^{z}, P} \vec{\nabla} T+\left(\frac{\partial H^{z}}{\partial m^{z}}\right)_{T, P} \vec{\nabla} m^{z} .
$$

Inserting this into the equation for the spin current (31), we obtain,

$$
\vec{J}_{m}^{z}=-\alpha\left(\frac{\partial H^{z}}{\partial m^{z}}\right)_{T, P}\left\{\vec{\nabla} m^{z}+\left(\frac{\partial m^{z}}{\partial H_{z}}\right)_{T, P}\left[\left(\frac{\partial H^{z}}{\partial T}\right)_{m^{z}, P}+\frac{\beta}{\alpha}\right] \vec{\nabla} T\right\} .
$$

Considering a magnetic fluid at rest $(\vec{v}=\overrightarrow{0})$, at constant temperature and at constant pressure from equation (35) the spin diffusion equation for the magnetization density per mass is given by

$$
\frac{\partial \mu_{m}}{\partial t}=\frac{\alpha}{\rho}\left(\frac{\partial H^{z}}{\partial \mu_{m}}\right)_{T, P} \nabla^{2} \mu_{m}
$$

Thus, the spin diffusion coefficient reads

$$
D_{m}=\frac{\alpha}{\rho}\left(\frac{\partial H^{z}}{\partial \mu_{m}}\right)_{T, P} .
$$

At vanishing spin current and at constant pressure, a temperature gradient causes a spin density gradient. From (44) we immediately obtain,

$$
\vec{\nabla} \mu_{m}=-\left(\frac{\partial \mu_{m}}{\partial H^{z}}\right)_{T, P}\left[\left(\frac{\partial H^{z}}{\partial T}\right)_{\mu_{m}, P}+\frac{\beta}{\alpha}\right] \vec{\nabla} T,
$$

which leads to the identification of the thermomagnetic diffusion ratio,

$$
\frac{k_{\mathrm{T}}}{T}=\left(\frac{\partial \mu_{m}}{\partial H^{z}}\right)_{T, P}\left[\left(\frac{\partial H^{z}}{\partial T}\right)_{\mu_{m}, P}+\frac{\beta}{\alpha}\right] .
$$

This transport coefficient corresponds to the thermodiffusion ratio in binary mixtures.

\section{Static functional of the Heisenberg fluid}

In order to find the static functional for the magnetic fluid near the gas-liquid phase transition we expand the general functional in the fluctuations of the densities $\alpha_{i}(x)$ per volume. The entropy density per volume represents the order parameter implying that the fluctuation terms of this 
density have to be taken into account up to the fourth order. The remaining terms in the static functional are the quadratic terms of all the other densities per volume mentioned and a quadratic gradient term for the order parameter. Because the order parameter is a scalar quantity, there is also a third order term allowed, consistent to the symmetry properties. In order to obtain a suitable static functional, several steps are necessary to be performed:

i) The densities per volume $\alpha_{i}(x)$ (except the mass density itself) will be replaced by densities per mass $\alpha_{i}(x) / \rho(x)$. The correlation functions will then be related to thermodynamic derivatives at fixed pressure instead of fixed chemical potential.

ii) In order to decouple the order parameter from the secondary densities a shift in the densities per mass has to be performed. This shift eliminates the quadratic terms between the order parameter and secondary densities and the third order term of the order parameter leading to the usual $\phi^{4}$-model.

iii) For a discussion of the slow heat and magnetic diffusion modes only, it is not necessary to keep the fast sound mode in the considerations. In this case it is convenient to integrate out the mass density both in the static and in the dynamic functional. Consequently the assignment of the background parameters in the quadratic part of the static functional changes from thermodynamic derivatives at a fixed chemical potential to corresponding quantities at a fixed pressure.

In the following we will consider the slowest modes only. In this case from the momentum density, separated into the longitudinal component $\vec{j}_{1}$ defined by $\vec{\nabla} \wedge \overrightarrow{j_{1}}=\overrightarrow{0}$, and the transverse component $\vec{j}_{\mathrm{t}}$, defined by $\vec{\nabla} \cdot \vec{j}_{\mathrm{t}}=0$, only the transverse part has to be considered. This finally leads to the static functional in an external magnetic field

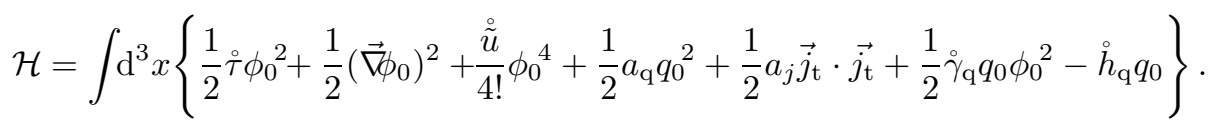

The fluctuations are defined as

$$
\begin{aligned}
\phi_{0}(x) & =\sqrt{N_{\mathrm{A}}}[\triangle \sigma(x)-\langle\sigma(x)\rangle], \\
q_{0}(x) & =\sqrt{N_{\mathrm{A}}}\left[\triangle \mu_{m}(x)-\left(\frac{\partial \mu_{m}}{\partial \sigma}\right)_{H, P}(\triangle \sigma(x)-\langle\sigma(x)\rangle)\right] .
\end{aligned}
$$

$N_{\text {A }}$ denotes the Avogadro number. The nonzero correlations are those of the order parameter and the density $q_{0}$.

Introducing the magnetic susceptibility

$$
\chi_{\sigma, P}=\left(\frac{\partial \mu_{m}}{\partial H^{z}}\right)_{\sigma, P}
$$

the static correlation function and the corresponding vertex function $\stackrel{\circ}{\Gamma}_{q q}$ are

$$
\left\langle q_{0} q_{0}\right\rangle_{\mathrm{c}}=\frac{1}{\stackrel{\circ}{\Gamma}_{q q}}=\frac{R T}{\rho} \chi_{\sigma, P} .
$$

Thus, the vertex function contains the magnetic susceptibility, which behaves like $\left(\partial \mu_{m} / \partial H^{z}\right)_{\sigma, P} \sim$ $t^{-\alpha}$ in the asymptotic critical region. The coefficient $a_{\mathrm{q}}$ corresponds to constant background values of thermodynamic derivatives. In the present case it is given by

$$
a_{\mathrm{q}}=\frac{\rho}{R T \chi_{\sigma, P}^{(0)}}=\frac{1}{\stackrel{\circ}{\Gamma}_{q q}^{(0)}}
$$

where $\stackrel{\circ}{\Gamma}_{q q}^{(0)}$ represents the zeroth order of the vertex function in (53). 
Apart from the Gaussian terms of the transverse spin components, which do not contribute to the critical behavior, the static functional (49) is the same as those of binary liquid mixtures at the plait point [19]. All thermodynamic relations can be taken over from this system if one replaces the concentration $c$ and the chemical potential $\Delta$ of the liquid mixture by the spin density $\mu_{m}$ and the magnetic field $H^{z}$ of the magnetic liquid. Therefore the static critical behavior of the quantities in the magnetic liquid is the same as the static critical behavior of the corresponding quantities in the liquid mixture at the plait point.

\section{Dynamical model for Heisenberg fluids near the gas-liquid critical point}

Using Zwanzig's projection operator method, one can derive dynamic equations that describe the time evolution of slow densities (see [31, 32]). Considering a set $\left\{\alpha_{k}(x, t)\right\}$ of slow densities, one obtains,

$$
\frac{\partial \alpha_{i}(x, t)}{\partial t}=V_{i}\left(\left\{\alpha_{k}(x, t)\right\}\right)-\sum_{j} \Lambda_{i j} \frac{\delta \mathcal{H}\left(\left\{\alpha_{k}(x, t\}\right)\right.}{\delta \alpha_{j}(x, t)}+\theta_{i}(x, t)
$$

with $\Lambda_{i j}=-L_{i j} \nabla^{2}$ for conserved densities and $\Lambda_{i j}=L_{i j}$ for non-conserved densities. The term,

$$
V_{i}\left(\left\{\alpha_{k}(x, t)\right\}\right)=\int \mathrm{d} x^{\prime} \sum_{j}\left[Q_{i j}\left(x, t ; x^{\prime}, t^{\prime}\right) \frac{\delta \mathcal{H}\left(\left\{\alpha_{k}(x, t\}\right)\right.}{\delta \alpha_{j}(x, t)}-\frac{\delta Q_{i j}\left(x, t ; x^{\prime}, t^{\prime}\right)}{\delta \alpha_{j}(x, t)}\right]
$$

constitutes the reversible part of equations. The functions $Q_{i j}$ are determined by,

$$
Q_{i j}\left(x, t ; x^{\prime}, t^{\prime}\right)=k_{\mathrm{B}} T\left\{\alpha_{i}(x, t), \alpha_{j}\left(x^{\prime}, t^{\prime}\right)\right\}
$$

where the brackets $\{.,$.$\} denote generalized Poisson brackets and k_{\mathrm{B}}$ the Boltzmann constant. $\mathcal{H}$ represents a static functional. The coefficients $L_{i j}$ are related to the Onsager coefficients of hydrodynamic theory. The functions $\theta_{i}(x, t)$ include contributions of fast variables that will be considered as stochastic forces. Assuming that these stochastic forces $\theta_{i}(x, t)$ are determined by a Markovian process, the coefficients $\Lambda_{i j}$ fulfill the relations

$$
\left\langle\theta_{i}(x, t) \theta_{j}\left(x^{\prime}, t^{\prime}\right)\right\rangle=\Lambda_{i j} \delta\left(x-x^{\prime}\right) \delta\left(t-t^{\prime}\right)
$$

In the case of Heisenberg fluids, the densities $\left\{\alpha_{k}(x, t)\right\}$ are represented by hydrodynamic densities $s(x, t), \rho(x, t), m^{z}(x, t)$ and $\vec{j}(x, t)$ considered in section 3 . The generalized Poisson brackets (9) -(13) determine the reversible part (56), which is independent of the external magnetic field. The coefficients $L_{i j}$ are related to Onsager coefficients introduced in section 3 . They may be identified by comparing the general structure (55) of dynamic equations with the linearized hydrodynamic equations (34)-(37). In the present case one has

$$
\begin{aligned}
\frac{\partial s}{\partial t} & =k_{\mathrm{B}} \gamma \nabla^{2} \frac{\delta \mathcal{H}}{\delta s}+k_{\mathrm{B}} T \beta \nabla^{2} \frac{\delta \mathcal{H}}{\delta m}+V_{\mathrm{s}}\left(\left\{\alpha_{k}\right\}\right)+\theta_{\mathrm{s}} \\
\frac{\partial m^{z}}{\partial t} & =k_{\mathrm{B}} T \beta \nabla^{2} \frac{\delta \mathcal{H}}{\delta s}+k_{\mathrm{B}} T \alpha \nabla^{2} \frac{\delta \mathcal{H}}{\delta m^{z}}+V_{m^{z}}\left(\left\{\alpha_{k}\right\}\right)+\theta_{m}, \\
\frac{\partial \rho}{\partial t} & =V_{\rho}\left(\left\{\alpha_{k}\right\}\right), \\
\frac{\partial \vec{j}}{\partial t} & =k_{\mathrm{B}} T\left(\zeta^{(0)}+\frac{4}{3} \bar{\eta}^{(0)}\right) \nabla^{2} \frac{\delta \mathcal{H}}{\delta \overrightarrow{j_{1}}}+k_{\mathrm{B}} T \bar{\eta}^{(0)} \nabla^{2} \frac{\delta \mathcal{H}}{\delta \overrightarrow{j_{\mathrm{t}}}}+\vec{V}_{j}\left(\left\{\alpha_{k}\right\}\right)+\vec{\theta}_{j} .
\end{aligned}
$$


The reversible contributions determined by generalized Poisson brackets are

$$
\begin{aligned}
V_{\mathrm{s}}\left(\left\{\alpha_{k}\right\}\right)= & -k_{\mathrm{B}} T \vec{\nabla} \cdot\left(s \frac{\delta \mathcal{H}}{\delta \vec{j}}\right), \\
V_{m^{z}}\left(\left\{\alpha_{k}\right\}\right)= & -k_{\mathrm{B}} T \sum_{i} \nabla_{i} m^{z} \frac{\delta \mathcal{H}}{\delta j_{i}}, \\
V_{\rho}\left(\left\{\alpha_{k}\right\}\right)= & -k_{\mathrm{B}} T \vec{\nabla} \cdot\left(\rho \frac{\delta \mathcal{H}}{\delta \vec{j}}\right), \\
\vec{V}_{j}\left(\left\{\alpha_{k}\right\}\right)= & -k_{\mathrm{B}} T\left(s \vec{\nabla} \frac{\delta \mathcal{H}}{\delta s}+\rho \vec{\nabla} \frac{\delta \mathcal{H}}{\delta \rho}+m^{z} \vec{\nabla} \frac{\delta \mathcal{H}}{\delta m^{z}}\right) \\
& -k_{\mathrm{B}} T \sum_{i}\left(j_{i} \vec{\nabla} \frac{\delta \mathcal{H}}{\delta j_{i}}+\nabla_{i} \vec{j} \frac{\delta \mathcal{H}}{\delta j_{i}}\right) .
\end{aligned}
$$

The Onsager coefficients $\alpha, \beta, \gamma, \zeta^{(0)}$ and $\bar{\eta}^{(0)}$ have been introduced in (30)-(33). The superscript (0) indicates uncritical background values. The equation of mass conservation (61) does not contain dissipative terms (Onsager coefficients) or stochastic forces.

With the set of dynamic equations, a dynamic functional can be established using the method of [33]. Within this functional, the critical behavior of thermal modes as well as the sound mode may be determined. The mass density and longitudinal momentum density, which are necessary only if one wants to consider the critical behavior of the sound, can be integrated out of the dynamical functional. The remaining dynamic functional corresponds to a reduced set of dynamic equations including the entropy density, the magnetization density and the transverse momentum density only, and a reduced static functional which is identical to (49) presented in the previous section. Introducing the order parameter (50) and the secondary density (51), the set of reduced dynamic equations reads

$$
\begin{aligned}
\frac{\partial \phi_{0}}{\partial t} & =\stackrel{\circ}{\Gamma} \nabla^{2} \frac{\delta \mathcal{H}}{\delta \phi_{0}}+\stackrel{\circ}{L} \nabla^{2} \frac{\delta \mathcal{H}}{\delta q_{0}}-\stackrel{\circ}{g} \frac{\delta \mathcal{H}}{\delta \overrightarrow{j_{\mathrm{t}}}} \cdot \vec{\nabla} \phi_{0}+\theta_{\phi} \\
\frac{\partial q_{0}}{\partial t} & =\stackrel{\circ}{L} \nabla^{2} \frac{\delta \mathcal{H}}{\delta \phi_{0}}+\stackrel{\circ}{\mu} \nabla^{2} \frac{\delta \mathcal{H}}{\delta q_{0}}-\stackrel{\delta \mathcal{H}}{g} \frac{\vec{H}}{\delta \dot{j}_{\mathrm{t}}} \cdot \vec{\nabla} q_{0}+\theta_{q} \\
\frac{\partial \overrightarrow{j_{\mathrm{t}}}}{\partial t} & =\stackrel{\circ}{\lambda_{\mathrm{t}}} \nabla^{2} \frac{\delta \mathcal{H}}{\delta \overrightarrow{j_{\mathrm{t}}}}+\stackrel{\circ}{g}\left[\frac{\delta \mathcal{H}}{\delta \phi_{0}} \vec{\nabla} \phi_{0}+\frac{\delta \mathcal{H}}{\delta q_{0}} \vec{\nabla} q_{0}\right]-\stackrel{\circ}{g} \mathcal{T} \sum_{i}\left(j_{i} \vec{\nabla} \frac{\delta \mathcal{H}}{\delta j_{i}}+\nabla_{i} \vec{j} \frac{\delta \mathcal{H}}{\delta j_{i}}\right)+\vec{\theta}_{\mathrm{t}}
\end{aligned}
$$

The corresponding static functional $\mathcal{H}$ is given by (49). The kinetic coefficients appearing in (67) and (68) are related to the Onsager coefficients introduced in (30) and (31) by,

$$
\begin{aligned}
& \stackrel{\circ}{\Gamma}=\frac{R \gamma}{\rho^{2}}, \quad \stackrel{\circ}{L}=\frac{R T}{\rho^{2}}\left(\beta+\left(\frac{\partial \mu_{m}}{\partial \sigma}\right)_{H^{z}, P} \frac{\gamma}{T}\right), \\
& \stackrel{R}{\mu}=\frac{R T}{\rho^{2}}\left(\alpha-2\left(\frac{\partial \mu_{m}}{\partial \sigma}\right)_{H^{z}, P} \beta+\left(\frac{\partial \mu_{m}}{\partial \sigma}\right)_{H^{z}, P}^{2} \frac{\gamma}{T}\right) .
\end{aligned}
$$

The non-linear mode coupling is defined as

$$
\stackrel{\circ}{g}=\frac{R T}{\sqrt{N_{\mathrm{A}}}} .
$$

From power counting arguments we obtain the cut-off $(\Lambda)$ dimensions of kinetic coefficients which are $\stackrel{\circ}{\Gamma} \sim \Lambda^{0}, \stackrel{\circ}{L} \sim \Lambda^{1}, \stackrel{\circ}{\mu} \sim \dot{\circ}_{\mathrm{t}} \sim \Lambda^{2}$, and $\stackrel{\circ}{g} \sim \Lambda^{1+\epsilon / 2}$. Within the perturbation expansion it is convenient to introduce time scale ratios

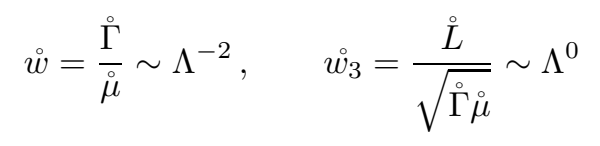


and a mode coupling parameter

$$
\stackrel{\circ}{\mathrm{t}}^{2}=\frac{\stackrel{\circ}{g}^{2}}{\stackrel{\circ}{\Gamma} \grave{\lambda}_{\mathrm{t}}} \sim \Lambda^{\epsilon}
$$

which has a finite fixed-point value. The time scale ratio $\stackrel{\circ}{w}$ is irrelevant as can be seen from its cut-off dimension and, therefore, has to be set to zero in expressions within perturbation expansion in order to obtain a theory renormalizable with minimal subtraction.

The dynamic equations (67) - (69) have the same form as in the case of binary liquid mixtures at the plait point [19]. Having considered the dynamical critical phenomena in the Heisenberg liquid, one can now proceed along the lines developed in [32, 33]. From the dynamical functional one calculates the dynamical correlations by a perturbation expansion in the mode couplings and the static couplings.

\section{Applying the field theoretic approach}

In order to proceed in the calculation of physical measurable quantities, e.g., the temperature dependence of a transport coefficient of the magnetic fluid, a standard procedure of field theory is followed. These steps are not repeated here. They are:

(i) A perturbational calculation (loop expansion) of the physical quantities.

(ii) The introduction of renormalized counterparts of unrenormalized static and dynamic model parameters presented so far leading to renormalization factors which contain the dimensional poles.

(iii) The calculation of the so-called $\zeta$-functions following from the renormalization factors, which determine the temperature behavior of the model parameters from the background to the asymptotic region (usually called the flow of parameters).

The transport coefficients in hydrodynamic limit (small frequencies and small modulus of the wave vector) are calculated from the vertex functions in this limit. In the asymptotic limit the result leads to power laws in the relative temperature distance from the gas-liquid critical point. The exponents are given by the $\zeta$-functions taken at the fixed point of the flow equations for kinetic coefficients or their ratios, and the static and dynamic couplings. The renormalized counterparts $\Gamma, L$ and $\mu$ of the kinetic coefficients fulfill the flow equations

$$
\ell \frac{\mathrm{d} \Gamma(\ell)}{\mathrm{d} \ell}=\Gamma(\ell) \zeta_{\Gamma}, \quad \ell \frac{\mathrm{d} L(\ell)}{\mathrm{d} \ell}=L(\ell)\left(-1+\zeta_{L}\right), \quad \ell \frac{\mathrm{d} \mu(\ell)}{\mathrm{d} \ell}=\mu(\ell)\left(-2+\zeta_{\mu}\right) .
$$

The flow parameter $\ell$ is related to the reduced temperature $t$ by the matching condition,

$$
\frac{\xi^{-2}(t)}{\left(\xi_{0}^{-1} \ell\right)^{2}}=1
$$

with the correlation length $\xi(t)$. The renormalized counterpart of the time scale ratio $\stackrel{\circ}{3}_{3}$ introduced in (73) is given by

$$
w_{3}(\ell)=\frac{L(\ell)}{\sqrt{\Gamma(\ell) \mu(\ell)}} .
$$

The $\zeta$-function $\zeta_{\Gamma}$, introduced in (75), can be separated into a static contribution and a purely dynamic part $\zeta_{\Gamma}^{(\mathrm{d})}$ [34]. The remaining two $\zeta$-functions $\zeta_{L}$ and $\zeta_{\mu}$ do not contain dynamic contributions. They fulfill the exact relations

$$
\zeta_{\Gamma}=\zeta_{\phi}+\zeta_{\Gamma}^{(\mathrm{d})}, \quad \zeta_{L}=\frac{1}{2} \zeta_{\phi}, \quad \zeta_{\mu}=0
$$

The static $\zeta$-function, $\zeta_{\phi}(u)$, depends only on the fourth order coupling of the $\phi^{4}$-model $u(\ell)$. It is obtained from renormalization of the $k^{2}$ terms of the static two point order parameter vertex 
function. Thus, a kinetic coefficient $\Gamma^{(\mathrm{d})}$ may be introduced which is obtained from $\Gamma$ by separating the static contributions. From (75), (77) and (78) it follows that the dynamic flow is determined by the equations

$$
\ell \frac{\mathrm{d} \Gamma^{(\mathrm{d})}(\ell)}{\mathrm{d} \ell}=\Gamma^{(\mathrm{d})}(\ell) \zeta_{\Gamma}^{(\mathrm{d})}, \quad \ell \frac{\mathrm{d} w_{3}(\ell)}{\mathrm{d} \ell}=-\frac{1}{2} w_{3}(\ell) \zeta_{\Gamma}^{(\mathrm{d})},
$$

with proper initial conditions. The functions $\zeta_{\phi}$ and $\zeta_{\Gamma}^{(\mathrm{d})}$ are calculated from the static and dynamic renormalization factors within the perturbation expansion. The renormalized counterpart of the

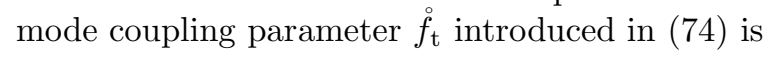

$$
f_{\mathrm{t}}^{2}(\ell)=\frac{g^{2}(\ell)}{\Gamma(\ell) \lambda_{\mathrm{t}}(\ell)}
$$

It is determined by the flow equation

$$
\ell \frac{\mathrm{d} f_{\mathrm{t}}(\ell)}{\mathrm{d} \ell}=-\frac{1}{2} f_{\mathrm{t}}(\ell)\left(\epsilon+\zeta_{\phi}+\zeta_{\Gamma}^{(\mathrm{d})}+\zeta_{\lambda_{\mathrm{t}}}\right) .
$$

The function $\zeta_{\lambda_{t}}$ is obtained from renormalization of the $k^{2}$ terms of transverse momentum current density two point function. The kinetic coefficient $\lambda_{t}(\ell)$ is determined by the flow equation,

$$
\ell \frac{\mathrm{d} \lambda_{\mathrm{t}}(\ell)}{\mathrm{d} \ell}=\lambda_{\mathrm{t}}(\ell)\left(-2+\zeta_{\lambda_{\mathrm{t}}}\right)
$$

In order to find the $\mathrm{FP}$ values of the time scale ratio $w_{3}$ one might use quite general arguments valid in all loop orders. Since the non-classical dynamical critical exponent of the OP is set by the FP value of $\zeta_{\Gamma}^{(\mathrm{d})}$ one expects its value nonzero and positive. Therefore it follows that the flow equation (79) drives $w_{3}$ to zero. Then at the FP all dynamical $\zeta$-functions reach their pure fluid form concerning the other parameters and thus from the FP equation for the mode coupling equation (81) one obtains the pure fluid value. Thus the asymptotic properties are the same as in pure fluids and the non-asymptotic properties are described by the same equations as for mixtures where concentration is replaced by magnetization in the direction of external field.

Let us note that this is not a general feature of mixtures. E.g., in mixtures of ${ }^{4} \mathrm{He}^{3}{ }^{3} \mathrm{He}$ at the superfluid transition, a similar time scale ratio $w_{3}$ appears involving only the kinetic coefficients of the conserved densities, entropy and concentration rather than the OP. In this case the FP value is nonzero [21].

\section{Critical behavior of the hydrodynamic transport coefficients}

In the previous section we have shown that the dynamic model for the Heisenberg fluid in an external homogeneous magnetic field reduces to the model known from binary liquid mixtures. The relations of hydrodynamic transport coefficients to the model parameters and their explicit calculation within renormalization group theory for a liquid mixture have been treated in [19]. Therefore, we shall not repeat these steps in the present paper, and only summarize the results we obtained.

From calculations analogous to those for binary liquid mixtures at the plait point [19], we obtain, at zero frequency, the thermal conductivity,

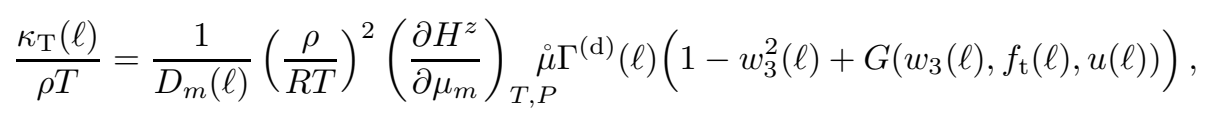

where $G\left(w_{3}, f_{\mathrm{t}}, u\right)$ contains contributions of perturbation expansion of the amplitude function for the dynamic order parameter two point function. In one loop order, one has $G\left(w_{3}, f_{\mathrm{t}}, u\right)=-f_{\mathrm{t}}^{2} / 16$. The spin diffusion coefficient $D_{m}$ appearing in (83) is given by,

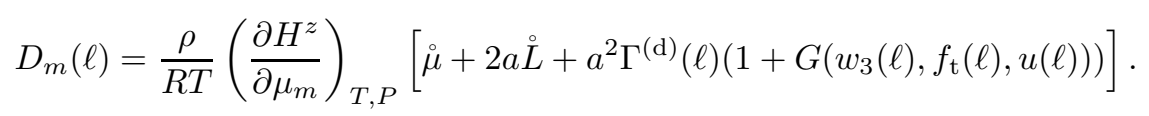


The parameter $a$ in the above equation is determined by thermodynamic derivative,

$$
a=\left(\frac{\partial \mu_{m}}{\partial \sigma}\right)_{H^{z}, P}
$$

which only weakly depends on the temperature and, therefore, can be considered as a constant in the critical region. The thermomagnetic diffusion ratio related to the cross effect between heat conduction and spin diffusion is,

$$
\frac{k_{\mathrm{T}}(\ell)}{T}=-\frac{\rho}{R T} \frac{1}{D_{m}(\ell)}\left[\stackrel{\circ}{L}+\frac{\stackrel{\circ}{a}}{a}\right]-\left(\frac{\partial \mu_{m}}{\partial T}\right)_{\sigma, P} .
$$

From the expressions (83), (84) and (86) for transport coefficients, the following power laws in the limit $\ell \rightarrow 0$ together with the matching condition (76) are found

$$
\kappa_{\mathrm{T}}(t) \sim \text { const. }, \quad D_{m}(t) \sim t^{\gamma-x_{\lambda} \nu}, \quad k_{\mathrm{T}}(t) \sim t^{-\gamma+x_{\lambda} \nu},
$$

where the exponents $\gamma, x_{\lambda}$ and $\nu$ can be taken from the renormalization theory for pure fluids (e.g. [21]). The value of the dynamic exponent in two-loop order reads $x_{\lambda}=0.9103$ [35].

The shear viscosity can be calculated as in pure fluids and liquid mixtures. At zero frequency the resulting expression for the shear viscosity is

$$
\bar{\eta}(\ell)=\frac{1}{R T}(\kappa \ell)^{2} \lambda_{\mathrm{t}}(\ell)\left(1+E_{\mathrm{t}}\left(f_{\mathrm{t}}(\ell), w_{3}(\ell)\right)\right) .
$$

The amplitude function $E_{\mathrm{t}}\left(f_{\mathrm{t}}(\ell), w_{3}(\ell)\right)$ contains contributions of perturbation expansion in the couplings (static and dynamic). In one loop order this function is $E_{\mathrm{t}}\left(f_{\mathrm{t}}(\ell), w_{3}(\ell)\right)=-f_{\mathrm{t}}^{2} /[36(1-$ $\left.w_{3}^{2}\right)$. The asymptotic temperature dependence is

$$
\bar{\eta}(t) \sim t^{-x_{\eta} \nu}
$$

with $x_{\eta}$ again taken from the renormalization group theory for pure fluids. In two loop order one obtains $x_{\eta}=0.0712$ [35]. The dynamical exponents fulfill the relation $x_{\eta}+x_{\lambda}=1-\eta$ with the two loop value of the static exponent $\eta=1 / 54$.

\section{Conclusion}

We have shown that the critical dynamics of a magnetic liquid in an external field is analogues to the critical dynamics of a liquid mixture at its plait point. The magnetization in the direction of the external field is then equivalent to the concentration of the mixture. In consequence, the corresponding transport coefficients behave in a similar manner although the non-asymptotic behavior might be different due to its different background values.

\section{Acknowledgments}

This work was supported by the Fonds zur Förderung der wissenschaftlichen Forschung under Project No. 19583-N20. 


\title{
References
}

1. Nijmeijer M.J., Weis J. J., Phys. Rev. E, 1996, 53, 591.

2. Nijmeijer M.J., ParolaA., Reatto L., Phys. Rev. E, 1998, 57, 465.

3. Schinagl F., Iro H., Folk R., Eur. Phys. J. B, 1999 8, 113.

4. Lado F., Lomba E., Phys. Rev. Lett., 1998, 80, 3535.

5. Lado F., Lomba E., Weis J.J., Phys. Rev. E, 1998, 58, 3478.

6. Sokoloska T. G., Sokolovskii R. O., Phys. Rev. E, 1999, 59, R3819.

7. Omelyan I.P., Mryglod I.M., Tokarchuck M.V., Phys. Rev. E, 1998, 57, 6667.

8. Hemmer P.C., Imbro D., Phys. Rev. A, 1977, 16, 380.

9. Omelyan I.P., Mryglod I.M., Folk R., Fenz W., Phys. Rev.E, 2004, 69, 061506.

10. Omelyan I.P., Fenz W., Mryglod I.M., Folk R., Phys. Rev. E, 2005, 72, 031506.

11. Folk R., Moser G., J. Phys. A: Math. Gen. 2006, 39, R207.

12. Kosterlitz J.M., Nelson D., Fisher M.E., Phys. Rev. B, 1976 ,13, 412.

13. Folk R., Holovatch Y., Moser G., Phys. Rev. E, 2009, 79, 031109.

14. Halperin B. I., Hohenberg P.C., Shang-keng Ma, Phys. Rev. B, 1974, 10, 139.

15. Folk R., Moser G., Phys. Rev. Lett. 2003, 91, 030601.

16. Halperin B.I., Hohenberg P.C. and Siggia E.D., Phys. Rev. B, 1976, 13, 1299; Halperin B.I., Hohenberg P.C., Siggia E.D., Phys. Rev. B, 1980, 21, 2044 (erratum).

17. De Dominicis D., Peliti L., Phys. Rev. B, 1978, 18, 353.

18. Pankert J., Dohm V., Phys. Rev. B, 1989, 40, 10842.

19. Folk R., Moser G., Phys.Rev.E, 1998, 58, 6246.

20. Folk R., Moser G., Phys.Rev.E, 2000, 61, 2864.

21. Folk R., Moser G., Phys.Rev.E, 1998, 57, 683, 705.

22. Folk R., Moser G., J.Low Temp.Phys., 1995, 99, 11.

23. Albrecht T., Bührer C., Fähnle M., Maier K., Platzek D., Reske J., Appl. Phys. A, 1997, 65, 215.

24. Brush G., Guentherodt H.J., Phys. Lett., 1968, 27A, 110.

25. Kraft B., Alexander H., Phys. Konden. Mat., 1973, 16, 281.

26. Dzyaloshinskii I.E., Volovick G.E., Ann.Phys.(N.Y.), 1980, 125, 67.

27. Mryglod I.M., Folk R., Physica A, 1996, 234, 129.

28. Mazur P., Non-Equilibrium thermodynamics, Dover Publ., New York 1984.

29. Zubarev D.N., Nonequilibrium Statistical Thermodynamics, Plenum Press, New York 1974.

30. Mryglod I.M., Tokarchyk M.V., Folk R., Physica A, 1995, 220, 325.

31. Graham R., Springer Tracts in Modern Physics 66, p.1 Springer, Berlin-Heidelberg-New York 1973.

32. Janssen H.-K., in Lecture Notes in Physics 104, p. 26 Springer, Berlin-Heidelberg-New York 1979.

33. Bausch R., Janssen H.-K., Wagner H., Z. Phys. B, 1976, 24, 113.

34. Folk R., Moser G., Phys. Rev. Lett. 2002, 89, 125301.

35. Adzhemyan L.Ts., Vasi'lev A.N., Kabrits Yu.S., Kompaniets M.V., Theor. and Math. Phys., 1999, 119, 454 .

\section{Стосовно класу універсальності магнітної рідини у зовнішньому полі}

\author{
Р. Фольк 1 , Г. Мозер² \\ 1 Інститут теоретичної фізики, Університет Йоганна Кеплера, Лінц, Австрія \\ 2 університет Зальцбурга, Зальцбург, Австрія
}

Коефіцієнти часових масштабів відіграють важливу роль у критичній динаміці. Значення їхніх фіксованих точок керують динамічною скейлінговою поведінкою полів, динамічними кореляційними функціями, а також гідродинамічними коефіцієнтами переносу. Як приклад системи з декількома коефіцієнтами часових масштабів ми розглядаємо магнітну рідину поблизу переходу газ-рідина.

Ключові слова: фазовий перехід, критична динаміка 